\title{
Sistema de monitoreo doméstico para consumo de agua
} Domestic monitoring system for water consumption

\author{
Janeth Alcalá-Rodríguez ${ }^{a}$, Juan C. Barbosa-García ${ }^{b}$, Manuel J. Contreras-Guzmán ${ }^{\text {, Jorge }}$ \\ Gudiño-Lau ${ }^{d}$, Saida M. Charre-Ibarra ${ }^{e}$, Daniel Vélez-Díaz ${ }^{f}$
}

\begin{abstract}
:
This paper proposes a system that allows real-time monitoring of total water domestic consumption by using wireless technology and through a developed user-friendly cellular phone application (APP). Results allow tracking and record the domestic water consumption. The proposal is constituted by a flow meter to monitor water consumption in the central point of the houseroom. Data are measured, collected, reported, and analysed by a control system that process the information and redirect the information via Bluetooth ${ }^{\circledR}$ to the APP. A database is included to register and save the history of the measured data to be consulted later by the user. The APP also sends reminders about water saving (when it is in use) and it is able to send an alert when the regular water domestic consumption is exceeded.
\end{abstract}

Keywords:

Water domestic consumption, water monitoring, water saving, wireless technology, cellular phone application

Resumen:

En este trabajo se propone un sistema que permite monitorear en tiempo real el consumo de agua total en una casa habitación utilizando tecnologías inalámbricas y que a través de una aplicación celular (APP) desarrollada de uso amigable permite llevar un seguimiento del consumo de agua. Los resultados permiten registrar y tasar el consumo de agua a nivel doméstico. El sistema se constituye por un medidor de flujo de caudal que monitorea el consumo de agua en la toma central de la casa habitación. Los datos son medidos, recogidos notificados y analizados por un sistema de control que procesa la información y la redirige vía Bluetooth ${ }^{\circledR}$ a la APP. Además, ofrece la opción enviar los datos monitoreados a un archivo en Excel, generando una base de datos que permite guardar un histórico de los datos medidos para posterior consulta por parte del usuario. La aplicación envía además (cuando está en uso) mensajes recordatorios sobre el cuidado del agua y puede emitir una alerta cuando se sobrepasa el umbral de consumo regular registrado en el hogar.

\section{Palabras Clave:}

Consumo de agua doméstico, monitor de agua, ahorro de agua, tecnología inalámbrica, aplicación celular

\section{Introducción}

El agua es uno de los recursos naturales más importantes para la vida del ser humano ya que satisface necesidades básicas del día a día, como es el aseo personal, la limpieza del entorno y principalmente, la hidratación del cuerpo. En las últimas décadas se ha observado una disminución o escases de este recurso natural que en muchos de los casos tiene que ver con una mala administración del recurso natural, perdidas debidas a fugas de agua o inclusive sistemas que no detectan las fugas y por ende no se genera registro de la perdida. De acuerdo a datos reportados, al día en México se consumen 380 litros de agua. Considerando que la población registrada en México (con datos cerrados al 2018) fue de 126.2 millones, se calcula que México

\footnotetext{
${ }^{a}$ Autor de Correspondencia, Universidad de Colima, https://orcid.org/0000-0002-0238-3952, Email: janethalcala@ucol.mx

${ }^{b}$ Universidad de Colima, https://orcid.org/0000-0002-7572-4252, Email: jgarcia159@ucol.mx

${ }^{c}$ Universidad de Colima, https://orcid.org/0000-0001-9836-4133, Email: mcontreras23@ucol.mx

${ }^{d}$ Universidad de Colima, https://orcid.org/0000-0002-0585-908X, Email: jglau@ucol.mx

${ }^{e}$ Universidad de Colima, https://orcid.org/0000-0002-3823-5388, Email: scharre@ucol.mx

${ }^{f}$ Universidad Autónoma del Estado de Hidalgo, https://orcid.org/0000-0001-6488-2960, Email: daniel@uaeh.edu.mx
} 
consume 47.956 litros de agua diariamente [1]. Para contextualizar, la OMS (Organización Mundial de la Salud), tiene establecido que el consumo diario debe ser de 100 litros de agua. Lo que convierte a México en un país de alto consumo, si bien no es que quien más consume, pues en los primeros puestos se encuentran China, India y USA, si es necesario sumar esfuerzos para atender esta problemática. Por ello, en los últimos años se han incrementado los esfuerzos a través de campañas de concientización del uso racionado y adecuado del agua; y se ha puesto mayor atención en desarrollar sistemas que permitan monitorear y preservar los recursos hídricos. Sobre todo, considerando que el $75 \%$ del planeta se compone de agua y este recurso llega a escasear en algunas regiones por lo cual, es razonable pensar en métodos para mejorar esta situación.

En relación a los sistemas de monitoreo, estos se pueden encontrar reportados y clasificados por 1) uso, ya sea en ambientes doméstico o industrial principalmente, o 2) por los tipos de sensores que utilizan.

Existen trabajos reportados en la literatura con fines comerciales que buscan como fin común el uso responsable y el cuidado del consumo de agua, como ejemplo, en [2]-[3] se abordan sistemas que consta de tres componentes principales; un nodo de interfaz para un medidor, un dispositivo de puerta de enlace y un sistema de backend. El nodo de interfaz del medidor se conecta a un medidor digital y cumple la función de recopilar las lecturas del medidor, gracias a un dispositivo gateway device (dispositivo de puerta de enlace) que envía las lecturas al backend. En el backend, los datos se procesan y archivan dentro de la base de datos utilizando Pandora como una aplicación de monitoreo, mientras que se desarrolla una herramienta visual para análisis basada en la web. O como el desarrollado en [4], que propone un medidor de agua para monitoreo remoto mediante comunicación inalámbrica ZigBee®.

Por otro lado, existen otras propuestas como la presentada en [5] que propone una forma más sencilla de medir el flujo de agua, por medio de un proceso que tiene como base identificar sitios de obstrucción y comparar las presiones de la zona obstruida y la libre, en la propuesta se remarca que esto puede realizarse sin necesidad de obstruir el ducto, incorporando tecnologías electromagnéticas para su lectura. En [6] se evalúa un medidor de agua basado en el internet de las cosas (IOT), este utiliza un medidor de agua ultrasónico STUF-280T que proporciona datos en protocolo M-bus y que utiliza una placa de desarrollo Linklt ONE para la comunicación entre el medidor de agua y la nube, utilizando Mediatek Cloud Sandbox para almacenar todos los datos dados por el medidor de agua.

A partir de los trabajos reportados en la literatura se pueden identificar una serie de técnicas y recomendaciones para medir el flujo de agua y cuantificar el consumo. Un punto clave en el que se coincide en los trabajos revisados apunta a que dependiendo el tipo de sistema que se desea monitorear se requiere adecuar el tipo de sistema que se puede instalar. En la propuesta que aborda el presente trabajo, se coloca en el punto central a un usuario común que no posea grandes conocimientos tecnológicos y que a través de un sistema sencillo y amigable pueda conocer el estado de su consumo, que pueda determinar los costos asociados a su consumo y que de acuerdo a la ingesta nominal de agua que suele tener se le pueda enviar una señal de alerta cuando se considera que esta se ha excedido. Para ello, es necesario identificar estudiar los parámetros, rangos, condiciones y tarifas con las que se evalúan y fijan las tarifas gubernamentales. Por lo que se propone diseñar un sistema que se adecue a las condiciones locales del municipio de Manzanillo, en el estado de Colima. La propuesta general contempla desarrollar un sistema que permite monitorear en tiempo real el consumo de agua total en el hogar utilizando tecnologías inalámbricas y que a través de una aplicación celular (APP) de uso amigable acceso libre permita llevar un seguimiento del consumo de agua por cualquier persona, con la posibilidad de registrar los datos medidos y tasar el consumo de agua a nivel doméstico. El sistema propuesto se constituye por un medidor de flujo de caudal que permite monitorear el consumo de agua en la toma central de una casahabitación, los datos medidos se envían a un sistema de control que se encarga de procesar y redireccionar los datos vía Bluetooth $\AA$ a una aplicación para el celular que se puede descargar e instalar fácilmente.

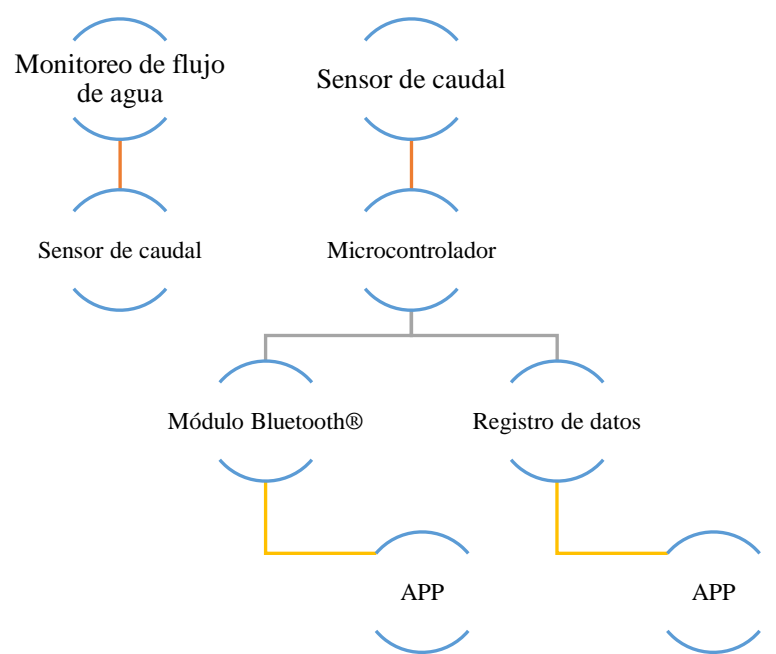

Figura 1. Diagrama para el sistema de monitoreo propuesto.

(Fuente: Elaboración propia). 
En la propuesta se incluye una base de datos que permite guardar un historial de los datos medidos para posterior consulta por parte del usuario. La aplicación envía además cuando está en uso mensajes recordatorios sobre el cuidado del agua y puede emitir una alerta cuando se sobrepasa el umbral de consumo regular registrado en el hogar como se muestra en la figura 1.

\section{Análisis del Sistema local CAPDAM}

CAPDAM (Comisión de Agua Potable, Drenaje y Alcantarillado de Manzanillo) es el organismo público descentralizado municipal que se encarga de operar y administrar los sistemas de agua potable, drenaje y alcantarillado de la zona metropolitana de Manzanillo. CAPDAM tiene un sistema de monitoreo y tarifario con el que se encarga de medir y fijar una cuota de acuerdo al consumo que tienes los habitantes de Manzanillo y clasifica a sus usuarios en 8 categorías que se pueden identificar con mayor detalle en [7] y que se resumen en la tabla 1.

Tabla 1. Clasificación y cuota tarifaria CAPDAM ${ }^{\star}$ [6].

\begin{tabular}{|l|c|}
\hline \multicolumn{1}{|c|}{ Clasificación } & Tarifa (MXN) \\
\hline $\begin{array}{l}\text { T-1: Uso doméstico en zona } \\
\text { rural e instituciones públicas y } \\
\text { de beneficencia pública y social. }\end{array}$ & $\$ 58.80$ Bimestral \\
\hline \begin{tabular}{l} 
T-2: Uso doméstico A \\
\hline T-3: Uso doméstico B
\end{tabular} & $\$ 70.80$ Bimestral \\
\hline $\begin{array}{l}\text { T-4: Uso condominal de carácter } \\
\text { privado }\end{array}$ & $\$ 137.76$ Bimestral \\
\hline $\begin{array}{l}\text { T-5: Uso Comercial "A" } \\
\text { T-6: Uso industrial o altos } \\
\text { consumos }\end{array}$ & $\$ 1948$ Bimestral \\
\hline \begin{tabular}{l} 
T-8: Uso Comercial "B" \\
\hline
\end{tabular} & $\$ 1458.72$ Mensual \\
\hline
\end{tabular}

Indistintamente de la clasificación, desde T-1 hasta T-8, los rangos de consumo se agrupan por $\mathrm{m}^{3}$ como se muestra en la tabla 2 para T-2.

En los recibos de agua que se entregan a la población bimestralmente por CAPDAM se puede ubicar información referente al usuario y su consumo, como se describe en los siguientes puntos:

- Fechas y cobros

- Datos generales de la entidad: Aquí muestran datos de la empresa encargado de dicho servicio.

- Contrato: Es el código del contrato.

- Periodo a pagar.

\footnotetext{
- En los casos que las tomas tengan un diámetro mayor de $1 / 2$ ", los usuarios pagarán la cantidad que la CAPDAM determine después de hacer el cálculo respectivo.
}

- Límite de pago: Es la fecha límite a pagar el pago de periodo.

- A pagar anual: Es el monto a pagar por los servicios anual mente.

- Límite anual: Es la fecha límite a pagar el pago anual.

- Periodo de la factura: Son las fechas que abarcan el cobro anual

- Datos del usuario: Muestra nombre, domicilio, entre otras cosas del propietario del hogar.

- CVE.CAT: Indica el código catastral, es el identificador oficial y obligatorio de los bienes inmuebles.

- Toma: muestra el tamaño de la toma de agua potable.

- Breve mensaje al usuario.

- Lectura anterior.

- Lectura actual.

- Metros cúbicos $\left(\mathrm{m}^{3}\right)$

- Datos de la ubicación del servicio (internos): Ruta, sector, servicio, código del medidor.

- Tipo de tarifa.

- Status del hogar.

- Historial de consumos.

- Estimado anual del año presente: Estimado por $\mathrm{m}^{3}$ y si es de tener uno, descuento, total a pagar.

- Una lista de adeudos.

\begin{tabular}{|c|c|c|}
\hline \multicolumn{3}{|c|}{ Rangos de consumo } \\
\hline De & Hasta & Tarifa (MXN) \\
\hline 0 & $30 \mathrm{~m}^{3}$ (rango mínimo) & $\begin{array}{c}\$ 70.80 \text { cuota } \\
\text { mínima bimestral }\end{array}$ \\
\hline 31 & $50 \mathrm{~m}^{3}$ & $\begin{array}{c}\$ 2.63 \text { por cada } \\
\text { metro cúbico }\end{array}$ \\
\hline 51 & $75 \mathrm{~m}^{3}$ & $\begin{array}{c}\$ 2.93 \text { por cada } \\
\text { metro cúbico }\end{array}$ \\
\hline 76 & $100 \mathrm{~m}^{3}$ & $\begin{array}{c}\$ 3.32 \text { por cada } \\
\text { metro cúbico }\end{array}$ \\
\hline 101 & en adelante $\mathrm{m}^{3}$ & $\begin{array}{c}\$ 7.09 \text { por cada } \\
\text { metro cúbico }\end{array}$ \\
\hline
\end{tabular}

Tabla 2. Rangos de consumo T-2: uso doméstico A [6].

De estos datos, se considera de interés y relevancia para mostrar en la APP que el usuario puede observar su consumo y su cuota mensual, además de verificar sus datos de facturación y reciba una alerta por exceso de consumo sobre el promedio bimestral. 


\section{Sistema de monitoreo}

Como se describió en la figura 1, la propuesta presentada en este trabajo está integra por un sistema sensor que se propone colocar en la toma central de agua en la casa habitación para medir el flujo de agua, y para lo cual se propone el sensor YF-S201 [8] que es un sensor de flujo o caudalímetro que permite medir el caudal o gasto volumétrico de un fluido. El caudal es la cantidad de líquido o fluido (volumen) que circula a través de una tubería por unidad de tiempo, por lo general se expresa en: litros por minutos $(\mathrm{L} / \mathrm{m})$, litros por hora $(\mathrm{L} / \mathrm{h})$, metros cúbicos por hora $\left(\mathrm{m}^{3} / \mathrm{h}\right)$. Los caudalímetros se colocan directamente en la tubería que transporta el fluido.

El sensor YF-S201 permite medir el caudal de agua en tuberías de $1 / 2^{\prime \prime}$ de diámetro y es un caudalímetro electrónico de tipo turbina, compatible con sistemas digitales como la tarjeta Arduino $\AA$, microcontroladores, tarjera Raspberry Pi y PLCs. El sensor posee tres cables: rojo (+5 V en $C D)$, negro (tierra) y amarillo (salida de pulsos del sensor de efecto Hall). En la tabla 3 se muestran sus especificaciones técnicas.

Tabla 3. Características técnicas del sensor de caudal YF-S201 [8].

\begin{tabular}{|c|c|}
\hline Parámetro & Valor \\
\hline Voltaje de operación & $+5-18 \mathrm{~V}$ en CD \\
\hline Consumo en corriente & $15 \mathrm{~mA}(\mathrm{a}+5 \mathrm{~V}$ en CD $)$ \\
\hline Capacidad de carga & $10 \mathrm{~mA}(\mathrm{a}+5 \mathrm{~V}$ en CD $)$ \\
\hline Salida & Onda cuadrada pulsante \\
\hline Rango del caudal & $1-30 \mathrm{~L} / \mathrm{min}$ \\
\hline Volumen promedio/pulso & $2.25 \mathrm{~mL}$ \\
\hline Pulsos/litro & 450 \\
\hline Factor de conversión & 7.5 \\
\hline Rosca externa & $1 / 2^{\prime \prime} \mathrm{NPS}$ \\
\hline Temperatura de operación & $-25^{\circ} \mathrm{C}$ a $80^{\circ} \mathrm{C}$ \\
\hline Material & $\begin{array}{c}\text { Plástico (recomendado para } \\
\text { evitar corrosión en } \\
\text { ambientes agresivos) }\end{array}$ \\
\hline
\end{tabular}

La frecuencia del caudal se pude calcular con la siguiente ecuación:

$f(H z)=K * Q(L / m i n)$

donde $\mathrm{K}$ es el factor de conversión y $\mathrm{Q}$ es el caudal en litros por minuto. $\mathrm{K}$ puede tomar los siguientes valores de acuerdo al tipo de rosca $K=(1 / 4 ": S),(1 / 2 ": 7,5),(3 / 4 ": 5,5)$, $(1 ": 4,8)$.

Los datos medidos se envían a una tarjeta embebida Arduino $\AA$, esta se encarga de recibir la frecuencia del sensor y mediante programación a partir de la ecuación 1 se obtiene el caudal por litros y posteriormente se traduce a volumen por litros y por metros cúbicos. De esta manera, en la tarjeta Arduino $\AA$ se registran los datos medios y se realiza los cálculos pertinentes al consumo y cuota mensual considerando los tabuladores mostrados en la tabla 2 (para uso doméstico). Posteriormente, se utiliza el módulo Bluetooth $\AA$ HC-05 [9], este módulo permite agregar conectividad inalámbrica a través de una interfaz serial TTL que proporciona Arduino $\circledast$ a un Smartphone. El módulo Bluetooth ${ }^{\circledR}$ HC-05 se configura mediante comandos AT y tiene la posibilidad de hacerlo funcionar tanto en modo maestro como esclavo, sus características técnicas se muestran en la tabla 4. Es importante resaltar en este punto que se optó por utilizar tecnología Bluetooth $\AA$ para enviar los datos a la APP propuesta y no a una red $\mathrm{WiFi}$, ya que se considera que, en promedio, el número de consumidores que cuentan con acceso a una conexión WiFi es reducido y por lo que está tecnología limitaría el uso del sistema propuesto en este trabajo a un grupo particular de la sociedad y el objetivo de este proyecto es que su uso sea de acceso libre, operable y gratuito para la sociedad en general.

Tabla 4. Características técnicas del Bluetooth ${ }^{\circledR}$ HC-05 [9].

\begin{tabular}{|c|c|}
\hline \multicolumn{1}{|c|}{ Parámetro } & Valor \\
\hline Voltaje de operación & $+3.6-6 \mathrm{~V}$ en CD \\
\hline Consumo en corriente & $50 \mathrm{~mA}$ \\
\hline Bluetooth & V2.0+EDR \\
\hline Frecuencia & Banda ISM $2.4 \mathrm{GHz}$ \\
\hline Modulación & GFSK \\
\hline Potencia de transmisión & $4 \mathrm{dBm}, \mathrm{Class} 2$ \\
\hline Alcance & $10 \mathrm{~m}$ \\
\hline Interfaz de comunicación & $1200 \mathrm{bps}$ hasta $1.3 \mathrm{Mbps}$ \\
\hline Baudrate (por defecto) & $38400,8,1, \mathrm{n}$ \\
\hline Temperatura de trabajo: & $-20{ }^{\circ} \mathrm{C} \mathrm{a}+75^{\circ} \mathrm{C}$ \\
\hline
\end{tabular}

Para desarrollar la APP se utilizó MIT App Inventor® que es un entorno de desarrollo de software creado por Google para la elaboración de aplicaciones que operan con el sistema operativo Android® [10]. Se optó por esta herramienta ya que es gratuita, permite acceder fácilmente a la web. Entre sus desventajas se puede mencionar que por su simplicidad están limitadas, no obstante, permiten cubrir un gran número de necesidades y para los efectos de este proyecto son una opción adecuada.

La APP es el medio visual informativo para el usuario y muestra los principales datos de interés para llevar un registro del consumo de agua en un hogar. Como puntos a resaltar, en la propuesta se considera que la APP sea capaz de entablar comunicación con un dispositivo móvil inteligente o Smartphone, que pueda enviar una alerta de 
anomalías sobre los datos monitoreados y que su formato sea simple, amigable con el usuario y no algo complicado de usar.

Como primer punto, se diseña la interfaz para el inicio de sesión, considerando datos como el nombre y generación de contraseña (cuando se registra por primera vez), para que en caso de que esta sea negativa no permita acceder a la pestaña siguiente, ya que contendrá información del usuario en particular. En la figura 2 se muestra el código de programación a bloques y en la figura 3 se muestra la interfaz propuesta.

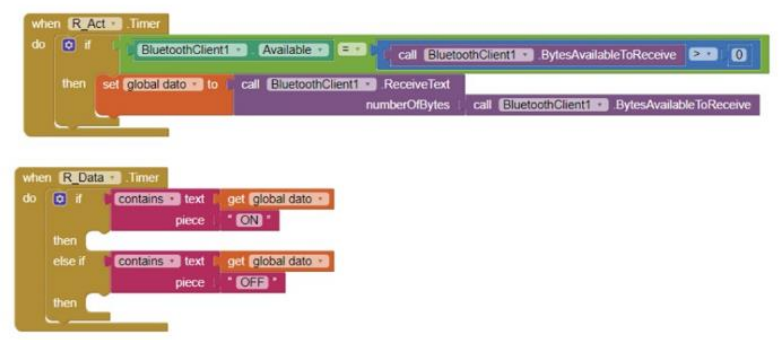

Figura 2. Estructura de programación para desarrollar la interfaz de inicio de sesión en MIT App Inventor $\AA$. (Fuente: Elaboración propia).

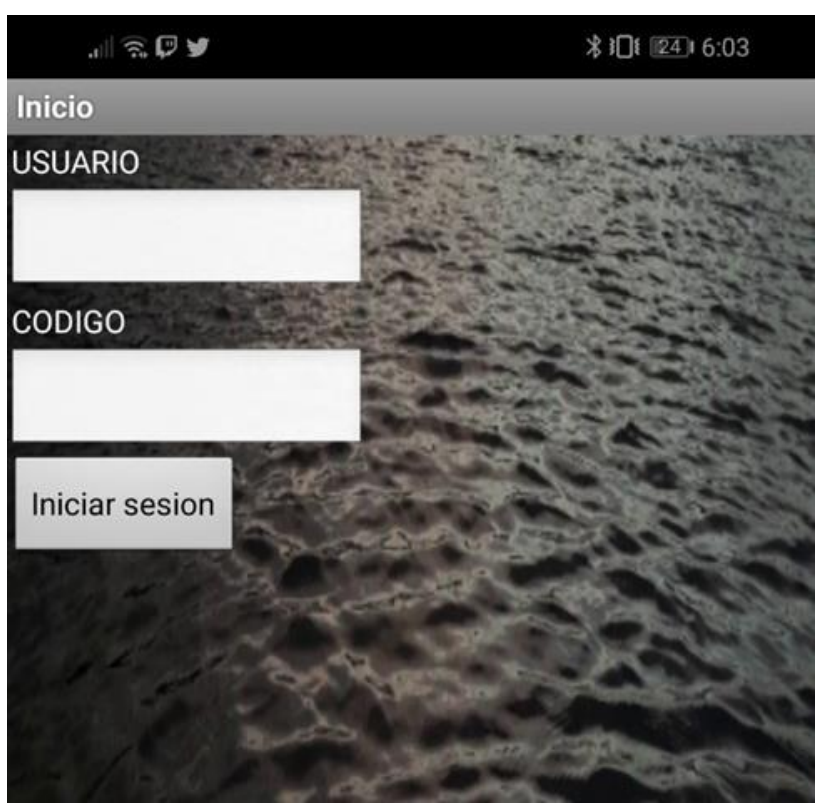

Figura 3. Interfaz desarrollada para inicio de sesión. (Fuente: Elaboración propia).

A continuación, se desarrolla la lógica para desplegar la información de interés para el usuario, es decir, la pestaña en donde el usuario puede ver toda su información, consumo y cuota mensual; y que además le permita verificar sus datos de facturación. En esta sección, es necesario agregar un botón para realizar la conexión con el módulo Bluetooth® HC-05. En la figura 4 se muestra el código de programación para esta sección. De igual forma, en la figura 5 se muestra la composición de bloques que realizan la acción de conectar y recibir datos provenientes del módulo Bluetooth $\AA \mathrm{HC}-05$, dando uso de bloques para toma de lista y bloques de búsqueda de cliente Bluetooth $\AA$, especificando que se reciba dato siempre y cuando este supere a 0 .

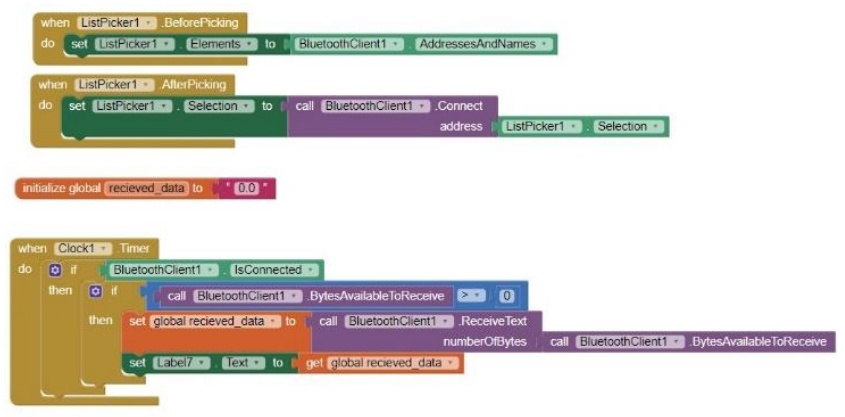

Figura 4. Estructura de programación para la sección de registro de datos.

(Fuente: Elaboración propia).

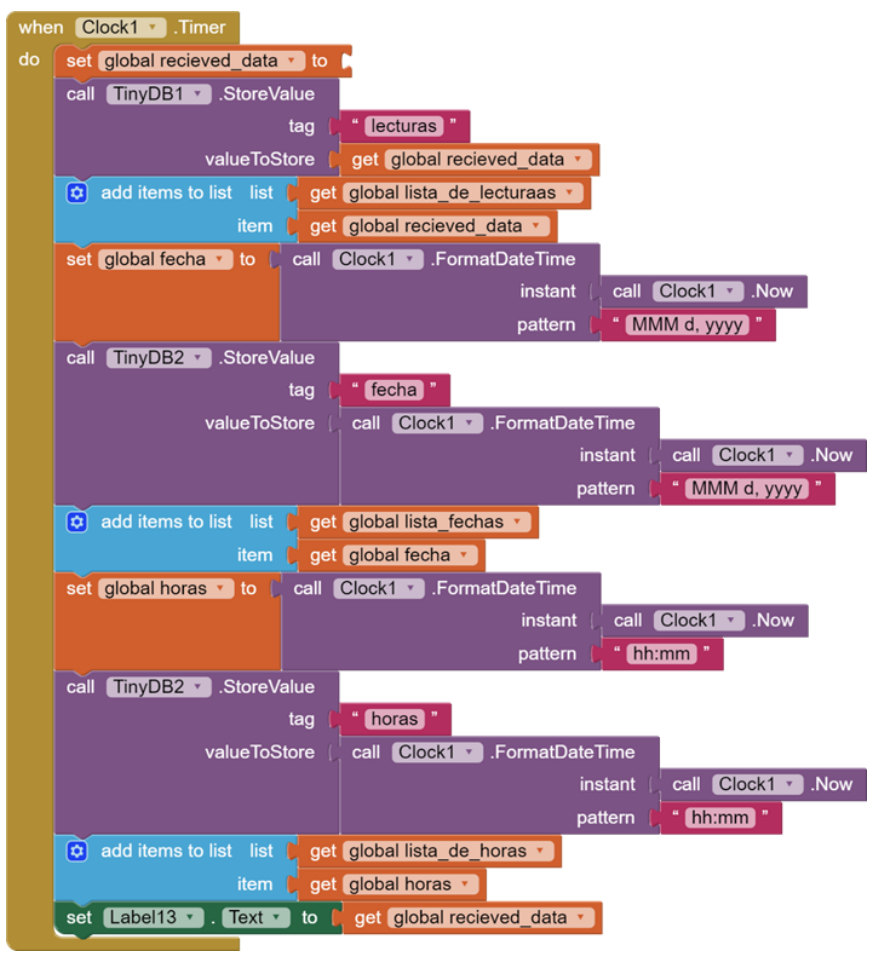

Figura 5. Estructura de programación conectar y recibir datos.

Fuente: Elaboración propia

Posteriormente, se aborda el campo de listas y almacenamiento de datos, para ellos se usan los bloques Make a list (donde el usuario realiza una lista y la nombra). Add items to list. Item (añade los datos que se desean enlistar para realizar la lista). Tiny $D B$ (base de datos para almacenar todos los datos que se desea encapsular). 
Además, se generan los bloques para adaptar, almacenar y relacionar cada dato, como se muestra en la figura 6.

Finalmente, se agrega el conjunto de bloques para la comparación y categorización de las lecturas utilizando los bloques and, if, then, math, string, entre otros. En este aspecto, se realizaron diferentes comparaciones respecto a una categorización de parte de CAPDDAM para calcular las cuotas.

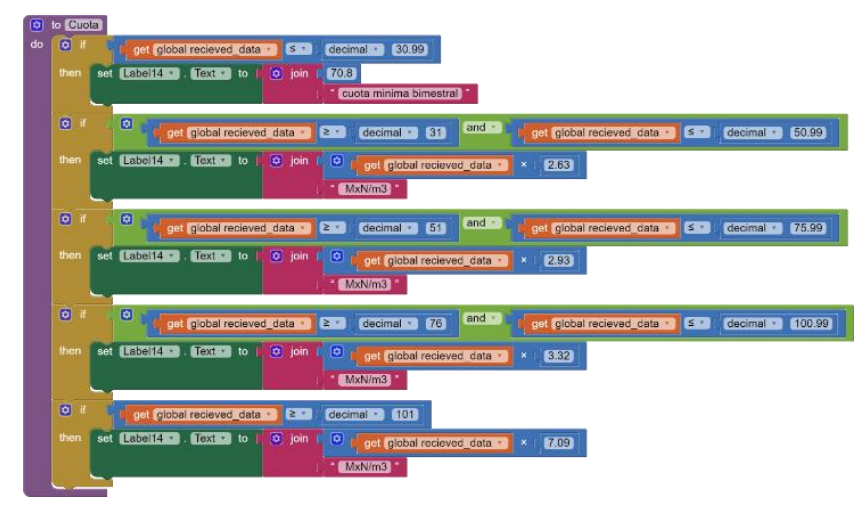

Figura 6. Estructura de programación para adaptar, almacenar y relacionar datos.

(Fuente: Elaboración propia).

En la figura 7 se muestra un esquema general a bloques de la propuesta descrita con la interfaz en operación.

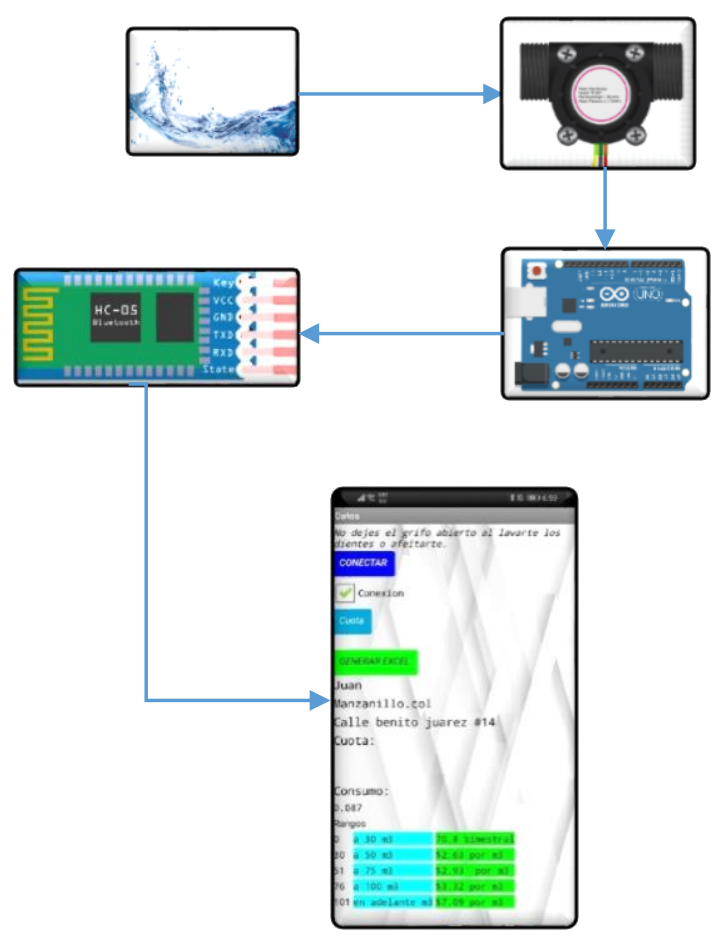

Figura 7. Esquema general operativo de la propuesta con la interfaz desarrollada operativa.

(Fuente: Elaboración propia).
En la figura 8 se muestra la aplicación instalada en el dispositivo móvil.

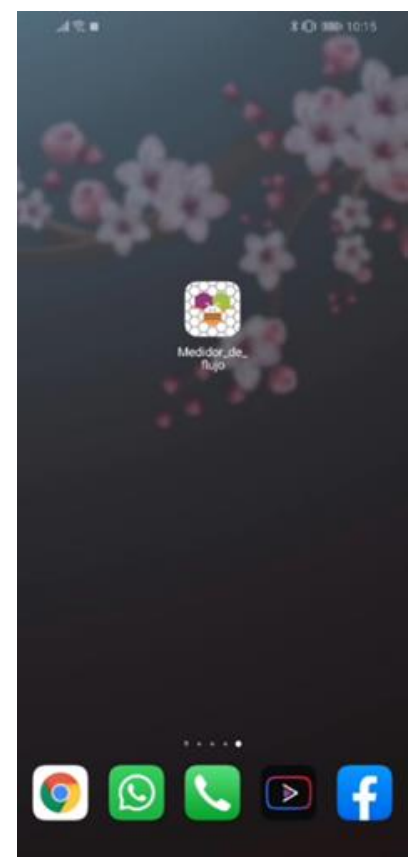

Figura 8. Aplicación desarrollada instalada en el dispositivo móvil.

(Fuente: Elaboración propia).

\begin{tabular}{|l|l|l|l|l|l|}
\hline \multicolumn{2}{|c|}{ Archivo Inicie Inser Disp Fórm } & Datc & Revis Vista & Ayuc Acro \\
\hline
\end{tabular}

Figura 9. Datos almacenados a partir de la lectura con la aplicación desarrollada.

(Fuente: Elaboración propia).

Se realizaron pruebas experimentales de consumo en una casa habitación, para efectos de tiempo, se ajustaron lecturas en un periodo de 1 a 3 horas para mostrar un registro corto de lecturas por efectos de espacio en el documento reporte. Gracias al botón de Cuota mostrado 
en la figura 7 , se puede determinar cuál es la cantidad monetaria acumulada al momento (referente al consumo), y con el botón de Generar Excel los datos monitoreados a lo largo del periodo se registrarán en un documento Excel en el dispositivo con los datos recabados por el periodo de uso (para mandar llamar la información es necesario que el usuario tenga instalada la aplicación para lectura de documentos en Excel). En la figura 9 se muestran los datos registrados para la prueba realizada.

En el documento en formato Excel se puede observar el consumo, la fecha y la hora de las lecturas, estas se registrarán cada hora, cabe resaltar que el documento no se actualizara solo, este se tiene que actualizar cada vez que el usuario requiera ver estos registros.

\section{Conclusiones}

En este trabajo se presentó una propuesta que contempla un sistema que permite monitorear en tiempo real el consumo total de agua en el hogar utilizando tecnologías inalámbricas y que a través de una aplicación desarrollada para un dispositivo móvil inteligente permite llevar el seguimiento del consumo de agua a nivel doméstico. Para la propuesta los datos monitoreados se adaptaron a las condiciones del municipio de Manzanillo en el estado de Colima, considerando los parámetros registrados por CAPDAM para el plano doméstico T-2. La aplicación permite conocer los datos de consumo, costo y llevar un registro de los datos medidos. Como trabajo futuro para dar seguimiento a este trabajo se desarrollará un análisis de confiabilidad operativa del sistema y se compararán los datos registrados con los proporcionados en los recibos que se emiten bimestralmente por CAPDAM.

\section{Referencias}

[1] Zaher R., Chaccour K. and Badr G., "Intelligent Software Simulation of Water Consumption in Domestic Homes," 2016 UKSim-AMSS 18th International Conference on Computer Modelling and Simulation (UKSim), 2016, pp. 99-104, doi: 10.1109/UKSim.2016.30.

[2] Ponce E., Leeb S. and Lindahl P., "Know the Flow: Non-Contact Magnetic Flow Rate Sensing for Water Meters," in IEEE Sensors Journal, vol. 21, no. 1, pp. 802-811, 1 Jan.1, 2021, doi: 10.1109/JSEN.2020.3014843.

[3] Mudumbe M. and Abu-Mahfouz A., "Smart water meter system for user-centric consumption measurement," 2015 IEEE 13th International Conference on Industrial Informatics (INDIN), 2015, pp. 993-998, doi: 10.1109/INDIN.2015.7281870.

[4] Anisha, R. A. Menon and A. Prabhakar, "Electronically controlled water flow restrictor to limit the domestic wastage of water," 2017 International conference on Microelectronic Devices, Circuits and Systems (ICMDCS), 2017, pp. 1-6, doi: 10.1109/ICMDCS.2017.8211591.

[5] Chakraborty S., Bera S., Mandal N., Marick S. and Chattopadhyay S., "A Novel Technique of Flow Measurement Using Pipe Line Pressure Head as a Measuring Parameter," 2017 International Conference on Computer, Electrical \& Communication Engineering (ICCECE), 2017, pp. 1-6, doi: 10.1109/ICCECE.2017.8526234.
[6] Cherukutota N. and Jadhav S., "Architectural framework of smart water meter reading system in IoT environment," 2016 International Conference on Communication and Signal Processing (ICCSP), 2016, pp. 0791-0794, doi: 10.1109/ICCSP.2016.7754253.

[7] Comisión de Agua Potable, Drenaje y Alcantarillado de Manzanillo. [En línea]. Disponible en: https://www.capdam.gob.mx/.

[8] Sensors \& Sensors Modules: YF-S201 Water Flow Sensor. [En línea]. Disponible en:https://html.alldatasheet.es/html-pdf/1221259/ETC1/YFS201/111/1/YF-S201.html.

[9] HC-05 Bluetooth to Serial Port Module. [En línea]. Disponible en: https://datasheetspdf.com/pdf/1418730/ITead/HC-05/1.

[10] MIT App Inventor. [En línea]. Disponible en: https://appinventor.mit.edu/. 